\title{
Baylor Scott \& White Health news
}

Baylor Scott \& White Health recognized by U.S. News \& World

Report as most awarded not-forprofit health care system in Texas

In the midst of great transformation in the health care field, Baylor Scott \& White Health remains committed to safe, quality care while working to drive down costs and improve each patient's experience. U.S. News \& World Report recognized that commitment in its 2019-2020 Best Hospitals list, and Baylor Scott \& White Health is the most awarded not-for-profit health care system in Texas for the second year in a row.

For the 2019-2020 rankings, U.S. News evaluated more than 4500 medical centers nationwide for 25 specialties, procedures, and conditions. In 16 specialty areas, 165 hospitals were ranked in at least one specialty, including two Baylor Scott \& White medical centers. Four of the system's hospitals were also recognized as "high performing" in at least one specialty, meaning that they were among the top $10 \%$ in the nation.

- Baylor Scott \& White The Heart Hospital-Plano is ranked \#27 in the nation for cardiology and heart surgery.

- Baylor University Medical Center is ranked \#45 nationally in gynecology. High-performing medical specialties recognized as being in the top $10 \%$ in the nation include gastroenterology and gastrointestinal surgery, nephrology, and orthopedics.

- Baylor Scott \& White Medical CenterTemple is recognized as high performing and among the top $10 \%$ in the nation for two specialties: nephrology and pulmonology and lung surgery.

- Baylor Scott \& White Medical CenterPlano is named high performing and among the top $10 \%$ in the nation in both gastroenterology and gastrointestinal surgery and pulmonology and lung surgery.

- Baylor Scott \& White Institute for Rehabilitation-Dallas earned a high performing rating and is among the top $10 \%$ in the nation for rehabilitation.

In rankings by state and metro area, U.S. News recognizes hospitals that excel across multiple areas of care, including specialty and common procedures and conditions. This year, U.S. News ranked Baylor Scott \& White's flagship academic medical centers in Dallas and Temple among the top 10 in the state: Baylor University Medical Center was ranked \#4 in Texas and Baylor Scott \& White Medical Center-Temple was ranked \#7 in Texas. In the Dallas-Fort Worth metro area, Baylor University Medical Center is ranked \#2 and Baylor Scott \& White All Saints Medical Center-Fort Worth is ranked \#5.

In total, U.S. News named 15 Baylor Scott \& White medical centers as "high performing" in at least one of nine possible common procedures and conditions.

\section{Baylor Scott \& White Health selected as 2019-2020 employer of excellence by Center for Healthcare Leadership and Management}

Baylor Scott \& White Health was selected as one of the American Academy

\section{UPCOMING CME PROGRAMS}

The A. Webb Roberts Center for Continuing Education of Baylor Scott \& White Health is offering the following programs:

- Pediatric Subspecialty for the Primary Care Provider, December 6, 2019, Flix Brewhouse, Round Rock, Texas

- Cardiology Update, December 7-8, 2019, Westin at the Domain, Austin, Texas For more information, visit https://www.bswhealth.med/cme.

of Physician Assistants Center for Healthcare Leadership and Management's winners of the 2019-2020 Employer of Excellence Awards. Baylor Scott \& White is the first health care organization in Texas to receive this honor and joins a group of health systems recognized across the nation. The award program is designed to showcase organizations that have implemented practices that create positive work environments for physician assistants (PAs) and encourage collaborative provider teams.

"Advanced practice professionals play an integral role in helping to meet our patients' health care needs," said Alejandro Arroliga, MD, MSc, FCCP, FACP, chief medical officer, Baylor Scott \& White. "These talented clinicians help reinforce our commitment to provide safe, quality care."

Criteria for the Employer of Excellence Awards focus on five key drivers: establishing a positive and supportive PA work environment; providing opportunities for PAs to provide meaningful input that leads to positive organizational change; keeping PAs informed about organizational activity and decisions; involving PAs in leadership efforts to improve the quality of patient care; and creating processes for effective conflict management.

"Our advanced practice professionals improve patient access and quality in a cost-effective way," said John Kasel, DHA, MS, PA-C, DFAAPA, director of Advanced Practice Professionals-Baylor Scott \& White Central Region. "Through the support of our organization, our physician assistants are helping to meet the growing care needs of patients we serve."

\section{Baylor Scott \& White Medical Center-Plano certified as comprehensive stroke center} Baylor Scott \& White Medical CenterPlano has received certification as a Level I 


\section{PHILANTHROPY NOTES}

\section{Positive purpose: Jeff York sets his mind to helping others}

Jeff York is a proponent of positive thinking. In his work and in life, Jeff talks about the importance of having a positive mental attitude and being in a peak emotional state. Out of a devastating cancer diagnosis, Jeff found not only new strength and purpose but also the connections he needed to bring his message of positivity to a group of people who need it most.

A few years ago, Jeff experienced unusual trouble swallowing. He visited his doctor and received test results that indicated that he was a fit, healthy 48-year-old. But an endoscopy that his doctor ordered the next week showed gastroesophageal junction adenocarcinoma. Physicians explained that it was already at stage IV.

Jeff received treatment in Houston, where he lived for months separated from his family while enduring chemotherapy, radiation, and, eventually, successful surgery. Learning that only one small tumor remained, Jeff prepared for a second round of treatments. He stayed positive until 21 new tumors were found 2 months later.

Jeff called his primary care physician in Dallas again to find the best oncologist for recurrences of his cancer. After 2 days, his doctor's research and recommendation brought him to Baylor Scott \& White Health. Several months later, following an aggressive treatment plan, Jeff received excellent news from his oncologist: His cancer was virtually undetectable.

Jeff asked how long he would need infusions of this drug, which he had been receiving every 2 weeks. The answer was that he would need to be on this medication for the rest of his life. He wanted so badly to be done with cancer and treatments, but he realized the opportunity he had before him: to help change the lives of others with a dose of positivity.

Jeff's next step was to become a credentialed Baylor Scott \& White volunteer. His caregivers talk about the positive light
Jeff brings to infusion rooms when he is there for treatments. From uplifting even the most downtrodden patients to honoring the passion and purpose of the dedicated oncology physicians and nurses, Jeff shares his mental management techniques and support with others in hopes of inspiring and enabling them as they make their way through the cancer journey. It's Jeff's personal form of ministry.

When Jeff heard about Baylor Scott \& White Health's partnership with the American Cancer Society to build a Hope Lodge on the campus of Baylor University Medical Center, he immediately wanted to be a part of it. He and his wife, Carmen, made a generous gift to help give hope a new home in Dallas.

"You can't believe the number of people who get sick and then their lives are in complete financial ruin," Jeff said. "You often need to live somewhere else during treatment, which can last for long periods of time, and many can't afford to do that. Hope Lodge is something absolutely critical一we need this."

"It's a gift," Jeff said of Hope Lodge. "It's a monumental move for thousands of people. It's not about the money; it's about what it does, and it's about drawing people together. It's about the hero's journey that all of these patients and caregivers take."

\section{Drawing on the strength of the human body for innovative clinical trials}

The cancer research and treatment centers of Baylor Scott \& White Health provide coordinated care across nearly every cancer and do so with a multidisciplinary team driven to incorporate advanced therapies and research into patient care. At the heart of the collaborative effort to bring innovative treatments to our patients is Ronan Kelly, MD, MBBCh, MBA, a medical oncologist, clinical researcher, and now one of the anchors of cancer care within the system.
As chief of oncology for the organization's North Texas cancer centers and medical director of oncology for Baylor Charles A. Sammons Cancer Center in Dallas, Dr. Kelly is making it his mission to position the Baylor Scott \& White Health cancer center network as a destination for patients seeking advanced immunotherapeutic treatments. Immunotherapy uses a patient's immune system to fight cancer. The response can often be much more durable than what is achieved with older chemotherapy regimens.

As a thoracic oncologist and former director of the Gastroesophageal Cancer Therapeutics Program at Johns Hopkins, Dr. Kelly developed an innovative portfolio of clinical trials in lung cancer and gastroesophageal cancer. He continues to lead numerous national and international phase II and phase III clinical trials investigating immunotherapeutic approaches and novel targeted drugs in both cancer types.

Dr. Kelly and his colleagues within the Charles A. Sammons Cancer Center are currently conducting clinical trials through Baylor Scott \& White Research Institute that are enrolling patients to investigate nextgeneration immunotherapeutics. Exciting results have come out of these clinical trials to date across a range of tumor types. For this reason, Dr. Kelly encourages patients to participate in research efforts whenever appropriate.

"Data has shown that patients can potentially do better on a clinical trial than with the standard treatment, but only $2 \%$ to $3 \%$ of American patients participate in clinical trials. We need to get that number up. That's how we're going to learn why some people respond to treatment and why some people do not, and learn to overcome that resistance. That's how we're going to beat cancer," he added.

For information on how you can support these or other initiatives at Baylor Scott \& White Health-North Texas, please contact Baylor Scott \& White Dallas Foundation at 214.820.3136. comprehensive stroke center by the Texas Department of State Health Services and Det Norske Veritas Healthcare USA. Baylor Scott \& White-Plano becomes one of two certified comprehensive stroke centers in Collin
County. With this certification, Baylor Scott \& White-Plano demonstrates the ability to provide quality care to treat the most complex stroke cases through evidence-based treatments. As a comprehensive stroke center, the medical center has the personnel, infrastructure, and expertise to diagnose and treat stroke patients who require intensive medical and surgical care, specialized testing, or interventional therapy. 


\section{RECENT GRANTS}

- Mandatory estimates of vaccine effectiveness against medically attended, PCR-confirmed influenza in West South Central USA Principal investigator: Manjusha Gaglani, MD

Sponsor: Centers for Disease

Control and Prevention

Funding: $\$ 1,300,000$

Award period: 8/1/2019-7/31/2020

- Core_Apt measure of PCR-based influenza vaccine effectiveness in inpatient adults

Principal investigator: Manjusha Gaglani, MD

Sponsor: Centers for Disease

Control and Prevention

Funding: $\$ 700,000$

Award period: 8/1/2019-7/31/2020

- GamePlan4Care: Web-based delivery system for REACH II

Principal investigator: Alan Stevens, $\mathrm{PhD}$

Sponsor: National Institutes of Health
Funding: $\$ 507,823$

Award period: 7/15/2019-5/31/2020

- Statewide evidence-based telehealth treatment

Principal investigator: Eric Meyer, $\mathrm{PhD}$

Sponsor: Texas Veterans

Commission

Funding: $\$ 500,000$

Award period: 7/1/2019-6/30/2020

- Reflux-induced epithelial-mesenchymal transition in benign Barrett's esopha-

gus

Principal investigators: Rhonda

Souza, MD, and Stuart Spechler, MD

Sponsor: National Institutes of Health

Funding: \$360,968

Award period: 9/1/2019-8/31/2020

- Closed-loop neuromodulation to treat PTSD

Principal investigator: Mark Powers, $\mathrm{PhD}$

Sponsor: University of Texas at
Dallas/Department of the Defense

Funding: $\$ 268,111$

Award period: 10/1/20189/30/2020

- Prolonged exposure therapy for posttraumatic stress disorder in spinal cord injury: A randomized controlled trial

Principal investigator: Mark Powers, $\mathrm{PhD}$

Sponsor: National Institute on

Disability, Independent Living, and

Rehabilitation Research

Funding: $\$ 199,492$

Award period: 9/30/20199/29/2020

- The Northern and Central Vietnam heart attack study

Principal investigator: Hoa Nguyen, MD, PhD, MS

Sponsor: National Institutes of Health

Funding: $\$ 160,293$

Award period: 5/1/2019-4/30/2020
As a Level I comprehensive stroke center, Baylor Scott \& White-Plano provides care for a variety of strokes: hemorrhagic or large ischemic strokes; aneurysmal subarachnoid hemorrhage; and strokes from unusual etiologies or requiring specialized testing or therapies such as endovascular surgery and those requiring multispecialty management. Additionally, the medical center serves as a resource for other local medical facilities, offering guidance and consultation for patient triage and availability of diagnostic tests or treatments. 